Y. M. Cho • H. D. Shin - B. L. Park · J. H. Kim •

K. S. Park $\cdot$ S. Y. Kim $\cdot$ H. K. Lee

\title{
Association between polymorphisms in the nuclear respiratory factor 1 gene and type 2 diabetes mellitus in the Korean population
}

Received: 16 November 2004 / Accepted: 2 April 2005 / Published online: 5 August 2005

(C) Springer-Verlag 2005

\begin{abstract}
Aims/hypothesis: Dysfunction in mitochondrial oxidative phosphorylation plays a central role in insulin resistance and type 2 diabetes. Nuclear respiratory factor 1 (NRF1) is a transcription factor that acts on nuclear genes encoding respiratory subunits and components of the mitochondrial transcription and replication machinery. Thus, we investigated its genetic association with type 2 diabetes. Methods: The NRF1 gene was sequenced to identify polymorphisms in 24 Korean DNA samples and then common variants were genotyped in 766 patients with type 2 diabetes and 303 non-diabetic subjects. Results: Twelve single nucleotide polymorphisms and one insertion/deletion polymorphism were identified. Six common variants among them were genotyped in a larger study. Although three individual polymorphisms appeared to be associated with type 2 diabetes ( $g .-46350$ insdel A, g. $+141 G>T$ and $g .+54529 A>G$ ), the effects were only marginal. However, a haplotype (H2) was associated with a decreased risk of type 2 diabetes and another haplotype $(H 4)$ was associated with an increased risk of type 2 diabetes ( $p$ values for the Haplo.Score test were 0.009 and 0.004 , respectively). Conclusions/interpretation: We demonstrated that two common haplotypes of NRF1 gene are associated with type 2 diabetes in the Korean population.
\end{abstract}

Y. M. Cho $\cdot$ J. H. Kim $\cdot$ K. S. Park $\cdot$ S. Y. Kim $\cdot$ H. K. Lee $(\bowtie)$ Department of Internal Medicine,

Seoul National University College of Medicine,

28 Yongon-dong, Chongno-gu,

Seoul, 110-744, South Korea

e-mail: hkleemd@snu.ac.kr

Tel.: +82-2-20722266

Fax: $+82-2-7657966$

Y. M. Cho · J. H. Kim - K. S. Park · H. K. Lee

Genome Research Centre for Diabetes and Endocrine Disease, Clinical Research Institute, Seoul National University Hospital, Seoul, South Korea

H. D. Shin · B. L. Park

Department of Genetic Epidemiology, SNP Genetics,

Seoul, South Korea
Keywords Mitochondria - Nuclear respiratory factor 1 . Oxidative phosphorylation - Polymorphism - Type 2 diabetes mellitus

Abbreviations FOXC1: fork head-related activator-3 . LD: linkage disequilibrium - NRF1: nuclear respiratory factor 1 - OR: odds ratio - PGC1: peroxisome proliferatoractivated receptor $\gamma$-coactivator $1 \cdot$ SNP: single nucleotide polymorphism - TFAM: mitochondrial transcription factor A - UTR: untranslated region

\section{Introduction}

Insulin resistance in the skeletal muscle of insulin-resistant offspring of type 2 diabetic patients is associated with decreased mitochondrial phosphorylation, which might be an inherited defect in mitochondrial oxidative phosphorylation [1]. Furthermore, age-associated insulin resistance also could be attributable to an age-associated reduction in mitochondrial oxidative phosphorylation capacity [2]. Therefore, dysfunction in mitochondrial oxidative phosphorylation might play an important role in the pathogenesis of insulin resistance and type 2 diabetes.

It has been suggested that the oxidative phosphorylation and electron transport chain system in mitochondria rely on the functional interplay of gene products expressed from both nuclear and mitochondrial genomes [3]. Since mitochondrial DNA encodes only 13 subunits of $\sim 100$ structural subunits of respiratory proteins and ATP synthase in the human mitochondria, the nucleo-mitochondrial interaction is essential to maintain normal cellular function [4]. Using microarray data, Patti et al. [5] and Mootha et al. [6] independently observed that the genes of oxidative metabolism regulated by nuclear-encoded proteins are coordinately reduced in the skeletal muscle of human subjects with insulin resistance or diabetes, which is in line with the fact that mitochondrial oxidative metabolism is crucial in maintaining normal insulin sensitivity and normal glucose metabolism [1, 2]. Interestingly, those genes downregulated in insulin resistance or diabetes are under the control 
of nuclear respiratory factor 1 (NRF1) and peroxisome proliferator-activated receptor $\gamma$-coactivator 1 (PGC1) $[5,6]$. Among them, NRF1 (Gene map locus 7q32; OMIM\# 600879 ) is a transcription factor that acts on nuclear genes encoding respiratory subunits and components of mitochondrial transcription and replication machinery [7, 8]. NRF1 binds to and activates the promoters of many genes of the mitochondrial electron transport system such as cytochrome $c$, NADH dehydrogenase subunit 8 , some cytochrome oxidase subunits, some ATP synthase subunits, mitochondrial transcription factor A (TFAM), and so on (reviewed in [9]). There is a growing body of evidence that links NRF1 physiologically to glucose metabolism. Aerobic exercise increases NRF1 expression in skeletal muscle [10] and muscle-specific overexpression of human NRF1 in transgenic mice increases glucose transport capacity in skeletal muscle by increasing glucose transporter 4 expression [11]. NRF1 mRNA expression in human skeletal muscle is decreased in diabetes and inversely correlated with fasting glucose [5]. In this regard, $N R F 1$ is an attractive candidate gene for type 2 diabetes. However, to the best of our knowledge, there is no study on the association between $N R F 1$ polymorphism and type 2 diabetes. Thus, in this study, we examined the genetic association between $N R F 1$ and type 2 diabetes.

\section{Subjects and methods}

\section{Subjects}

For initial sequencing, 24 Koreans were randomly selected from unrelated local residents without any history of familial diseases. With 24 samples, one can expect to identify more than $90 \%$ of polymorphisms with a frequency greater than 0.05 [12]. Subsequently, we studied 766 unrelated patients with type 2 diabetes from the Diabetes Clinic of Seoul National University Hospital (age: $59 \pm 10$ years, 357 men, 409 women) and 303 non-diabetic control subjects (age: $65 \pm 4$ years, 139 men, 164 women). The age of onset of diabetes was $50 \pm 10$ years. All subjects enrolled in this study were of Korean ethnicity. Type 2 diabetes was diagnosed according to World Health Organization criteria [13]. Subjects with positive GAD antibodies were excluded. Selection of the non-diabetic control subjects was according to the following criteria: 60 years or older, no past history of diabetes, no diabetes in first-degree relatives, a fasting plasma glucose concentration of less than $6.1 \mathrm{mmol} / \mathrm{l}$, and an $\mathrm{HbA}_{1} \mathrm{c}$ value of less than $5.8 \%$. This study was carried out in accordance with the Declaration of Helsinki as revised in 2000 (http://www.wma.net/e/policy/ 17 cnote.pdf).

The Institutional Review Board of the Clinical Research Institute in Seoul National University Hospital approved the study protocol and written informed consent for genetic analysis was obtained from each subject.

All study subjects were examined in the morning after an overnight fast. Height, weight, circumferences of waist and hip and blood pressure were measured. Blood samples were drawn for biochemical measurements (fasting plasma glucose, fasting plasma insulin, $\mathrm{HbA}_{1} \mathrm{c}$, total cholesterol, triglyceride and HDL-cholesterol) and DNA extraction.

Sequencing analysis of the human NRF1 gene

We sequenced all exons, including exon-intron boundaries and promoter region $(\sim 1.5 \mathrm{~kb})$, to discover polymorphisms in 24 Korean DNA samples using the ABI PRISM 3700 DNA Analyzer (Applied Biosystems, Foster City, CA, USA). Seventeen primer sets for the amplification and sequencing analysis were designed based on GenBank sequences (NM_005011). Information regarding the primers is available at http://www.snp-genetics.com/reference/ NRF1_add_info.doc. Sequence variants were verified by chromatograms.

Genotyping with fluorescence polarisation detection

For genotyping of polymorphic sites, amplifying primers and probes were designed for TaqMan. Primer Express (Applied Biosystems) was used to design both the PCR primers and the MGB TaqMan probes. One allelic probe was labelled with the FAM dye and the other with the fluorescent VIC dye. PCRs were run in a TaqMan Universal Master mix without UNG (Applied Biosystems) with PCR primer concentrations of $900 \mathrm{nmol} / \mathrm{l}$ and MGB TaqMan probe concentrations of $200 \mathrm{nmol} / \mathrm{l}$. Reactions were performed in a 384-well format in a total reaction volume of $5 \mu \mathrm{l}$ using $20 \mathrm{ng}$ genomic DNA. The plates were then placed in a thermal cycler (PE 9700; Applied Biosystems) and heated at $50^{\circ} \mathrm{C}$ for $2 \mathrm{~min}$ and $95^{\circ} \mathrm{C}$ for $10 \mathrm{~min}$ followed by 40 cycles of $95^{\circ} \mathrm{C}$ for $15 \mathrm{~s}$ and $60^{\circ} \mathrm{C}$ for $1 \mathrm{~min}$. The TaqMan assay plates were transferred to a Prism 7900HT instrument (Applied Biosystems) where the fluorescence intensity in each well of the plate was read.

Fluorescence data files from each plate were analysed by automated software (SDS 2.1). Genotyping quality control was performed in $10 \%$ of samples by duplicate checking (rate of concordance in duplicates $>99 \%$ ). The genotype success rate was $>97.5 \%$. Information regarding the primers is available on our website http://www.snp-genetics. com/reference/NRF1 add info.doc.

\section{Statistical analyses}

We used chi-square tests to determine whether individual polymorphisms were in Hardy-Weinberg equilibrium. Logistic regression analyses were used for calculating odds ratio (ORs), 95\% CIs and corresponding $p$ values, controlling for age, sex and BMI as covariates. Genotypes were given codes of 0,1 and 2 and 0,1 and 1 or 0,0 and 1 in the additive, dominant or recessive models, respectively. In the additive model, the OR was expressed per difference in number of rare allele. Multiple regressions, whilst adjusting 
for age and sex, were used for association analyses of diabetes-related phenotypes. Haplotype associations were also estimated using Haplo.Score (http://www.biostat.wustl. edu/genetics/geneticssoft), which imputes score statistics to test associations between haplotypes and a wide variety of traits, including binary, ordinal, quantitative and Poisson [14]. This software provides several haplotype-specific tests for association, as well as adjustment for non-genetic covariates and computation of simulation $p$ values, using the assumption that all subjects are unrelated and that haplotypes are ambiguous due to unknown linkage phases of the genetic markers. To predict putative transcription factor binding sites, we used MatInspector (http://www.genoma tix.de/products/MatInspector/index.html) [15]. A $p$ value of less than 0.05 was considered statistically significant.

\section{Results}

Identification of polymorphisms in the NRF1 gene

In this study, the NRF1 gene including all exons, $\pm 50 \mathrm{bp}$ exon-intron boundaries and the $-1,500$ bp 5'-flanking region were sequenced in 24 Korean DNA samples and 12 single nucleotide polymorphisms (SNPs) and one insertion/ deletion polymorphism were identified (Fig. 1a). Five variants were in the $5^{\prime}$ region: $g .-46656 G>A, g .-46568 G>A$, g.-46350insdel $A, g .-46185 G>C$, and $g .-45903 C>T$. Two SNPs were found in the exons, where both predicted silent substitutions: $g .+141 G>T$ in exon 2 encoding 47Ser and $g .+33162 C>T$ in exon 5 encoding 191Asp. Three SNPs were in introns: $g .+14382 T>C, g .+54529 A>G$, and $g .+59745 A>G$. And three SNPs were in the 3 -untranslated region (UTR) $(g .+97884 G>A, g .+97893 G>T$ and $g .+98560 A>G)$. By pair-wise linkage analysis, we have found that five sets of SNPs $(g .-46568 G>A: g .-46185 G>C, g .-46568 G>A$ : g. $-45903 C>T, g .-46185 G>C: g .-45903 C>T, g .+141 G>T$ : g. $+54529 A>G, g .+14382 T>C: g .+97893 G>T)$ were in absolute linkage disequilibrium (LD) $\left(\mathrm{LD} D^{\prime}=1\right.$ and $r^{2}=1$ ) (see Fig. 1a). Among identified polymorphisms, six common variants $(g .-46568 G>A, g .-46350 i n s d e l$ A, g.+ $141 G>T$, $g .+33162 C>T, g .+54529 A>G$ and $g .+59745 A>G)$ were selected for larger scale genotyping. The LD patterns did not show a break among SNPs genotyped (all $D^{\prime}$ s are $>0.9$, see Fig. 1b). Genotype distributions of all loci in NRF1 were in Hardy-Weinberg equilibrium $(p>0.05)$.

\section{a Map of NRF1 on chromosome 7q32}

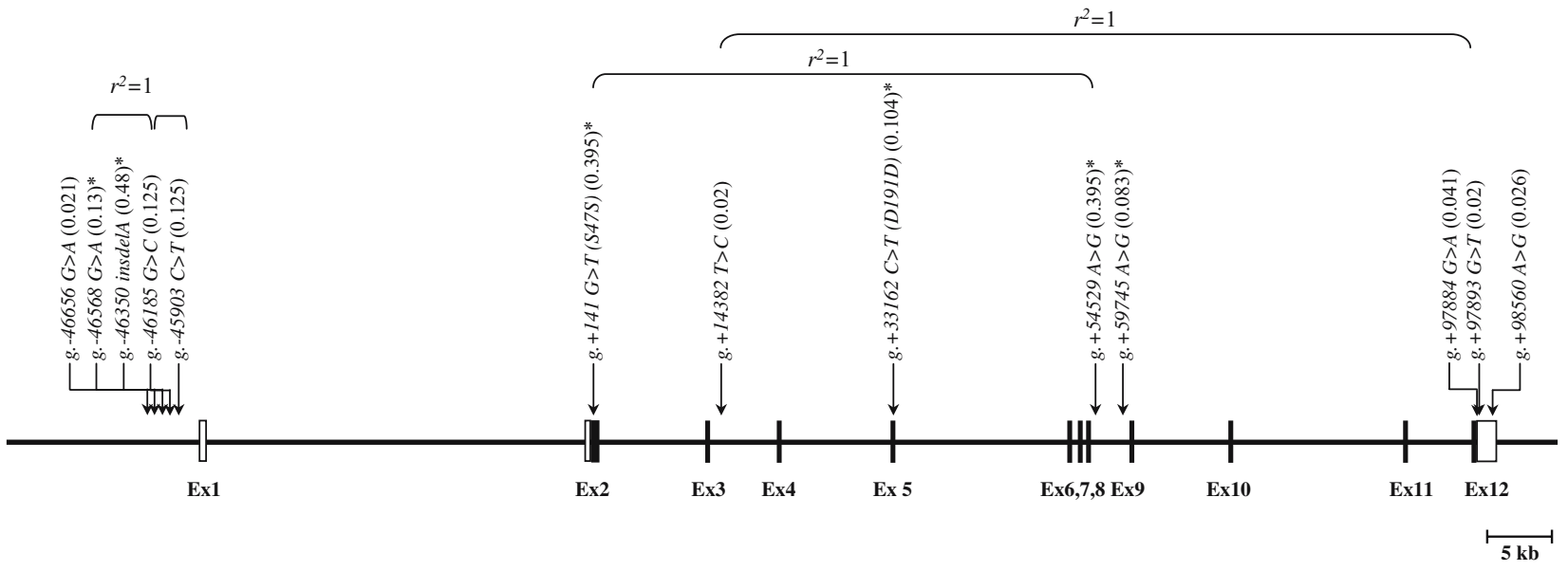

b LDs among NRF1 polymorphisms

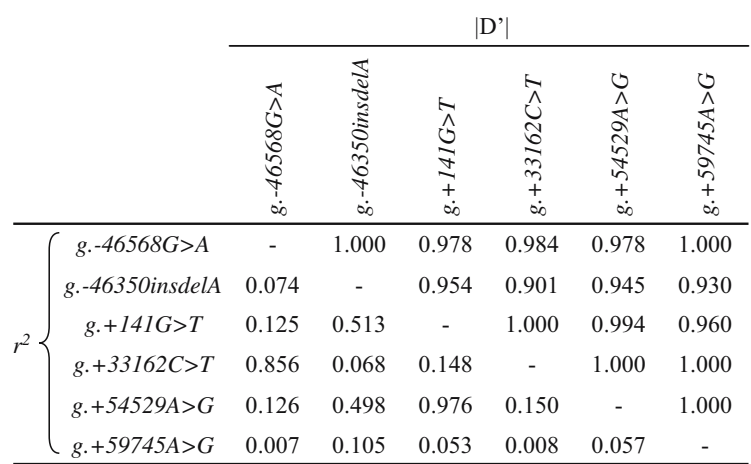

Fig. 1 Gene map (a) and LD pattern (b) of the NRF1 gene. a Polymorphisms identified in $N R F 1$, with coding exons marked by closed blocks and $5^{\prime}$ - and 3 '-UTRs by open blocks. ${ }^{*}=$ SNPs that were genotyped in the larger population. The frequencies of poly- morphisms were based on sequencing data $(n=24)$. The first nucleotide of the translation start site is denoted as nucleotide +1 . b LD coefficients $\left(\left|D^{\prime}\right|\right.$ and $\left.r^{2}\right)$ among polymorphisms in NRF1 
Association with type 2 diabetes

We assessed the association of each genotype by logistic regression analyses adjusting for age, sex and BMI. Three individual polymorphisms were found to be associated with the risk of type 2 diabetes (Table 1). The g.-46350insdel $A$ showed an increased risk of type 2 diabetes when applying a recessive genetic model $(\mathrm{OR}=1.631,95 \%$ CI 1.139-2.337, $p=0.008)$. Also, the nucleotide position $-46,350$ was predicted as a 3'-flanking site of the fork head-related activator-3 (FOXC1) consensus sequence. The $g .+141 G>T$ showed an increased risk of type 2 diabetes with an additive model $(\mathrm{OR}=1.341[1.075-1.671], p=0.009)$ and with a dominant model $(\mathrm{OR}=1.396$ [1.050-1.855], $p=0.022)$. The $g .+54529 A>G$ polymorphism, which is in complete
LD with $g .+141 G>T$, showed an increased risk of type 2 diabetes with an additive model $(\mathrm{OR}=1.302$ [1.046-1.619], $p=0.018)$ and with a dominant model $(\mathrm{OR}=1.398$ [1.0531.857], $p=0.021)$. When Bonferroni's correction for the multiple comparisons was strictly applied, no polymorphism remained significant in terms of the association with type 2 diabetes.

Among the six different polymorphisms genotyped, we identified five common haplotypes (frequency $>0.05$ ) that account for $97.6 \%$ of the observed haplotypes (Table 2). The haplotype $H 2$ was associated with a decreased risk of type 2 diabetes ( $p$ value for Haplo.Score $=0.009$ ) and the haplotype $H 4$ was associated with an increased risk of type 2 diabetes ( $p$ value for Haplo.Score=0.004). Comparing the elements of the protective haplotype $H 2$ and the sus-

Table 1 Associations between polymorphisms in NRF1 and the risk of type 2 diabetes

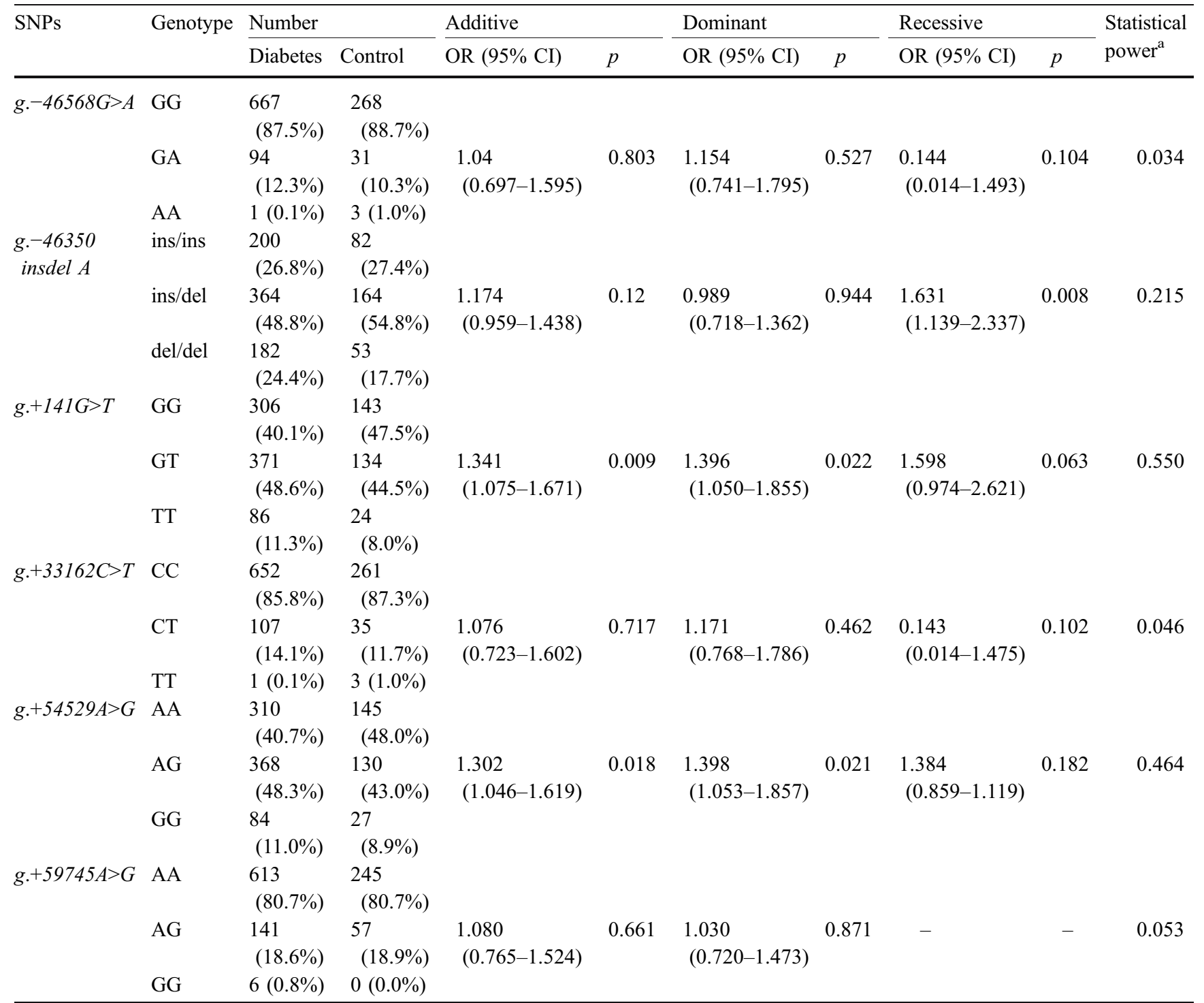

Genotype distributions are shown as number (\%). Odds ratios (ORs), 95\% CI, and $p$ values were from logistic regression analyses with additive, dominant, and recessive models controlling for age, sex and BMI as covariates. In additive models, ORs are expressed per difference in number of rare alleles. $p$ values were not corrected for multiple comparisons (18 tests)

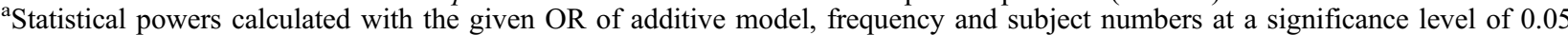


Table 2 Association between haplotypes and the risk of type 2 diabetes

\begin{tabular}{|c|c|c|c|c|c|c|c|c|c|}
\hline \multirow[t]{2}{*}{ Haplotype } & \multicolumn{6}{|l|}{ Locus } & \multicolumn{2}{|c|}{ Frequency } & \multirow[t]{2}{*}{$p$ value } \\
\hline & $-46,567$ & $-46,350$ & 141 & 33,162 & 54,529 & 59,745 & Diabetes & Control & \\
\hline$H 1$ & G & insA & $\mathrm{G}$ & $\mathrm{C}$ & A & A & 0.499 & 0.533 & 0.138 \\
\hline$H 2$ & G & $\operatorname{del} A$ & $G^{b}$ & $\mathrm{C}$ & $A^{b}$ & A & 0.042 & 0.066 & 0.009 \\
\hline$H 3$ & G & $\operatorname{del} A$ & G & $\mathrm{C}$ & A & $\mathrm{G}$ & 0.097 & 0.087 & 0.626 \\
\hline H4 & $\mathrm{G}$ & delA & $\mathrm{T}^{\mathrm{b}}$ & $\mathrm{C}$ & $\mathrm{G}^{\mathrm{b}}$ & A & 0.277 & 0.226 & 0.004 \\
\hline H5 & $\mathrm{A}$ & $\operatorname{delA}$ & $\mathrm{T}$ & $\mathrm{T}$ & $\mathrm{G}$ & A & 0.062 & 0.061 & 0.880 \\
\hline
\end{tabular}

Each haplotype with a frequency $>0.05$ is shown

${ }^{a} p$ values of haplotype associations were calculated by the Haplo.Score algorithm developed by Schaid et al. [14], while controlling for age, sex and BMI as covariates. They were not corrected for multiple comparisons (five tests)

${ }^{\mathrm{b}}$ Alleles regarded as the determinants modifying susceptibility to type 2 diabetes after examining the allelic differences between the protective (H2) and susceptible (H4) haplotypes

ceptible haplotype $H 4$, two polymorphisms $(g .+141 G>T$ and $g .+54529 A>G$ ) were regarded as the determinants modifying the risk of type 2 diabetes, although their individual effect was modest, as shown in Table 1.

\section{Association with diabetes-related phenotypes}

For the association analyses of the diabetes-related phenotypes, only non-diabetic subjects were used because, in diabetic subjects, treatment of diabetes might have affected those parameters. However, analyses of fasting plasma glucose, homeostasis model assessment of insulin resistance, waist circumference, BMI, total cholesterol, triglyceride and HDL-cholesterol did not reveal any strong associations with genotypes or haplotypes when adjusting for multiple comparisons (data not shown). Also, there were no strong associations between the diabetes-related phenotypes and the protective haplotype $H 2$ or the susceptible haplotype $H 4$ (Table 3 ).

\section{Discussion}

In this study, we demonstrated that two common haplotypes of the NRF1 gene are associated with type 2 diabetes in the Korean population. To the best of our knowledge, this is the first study to demonstrate that NRF1 polymorphisms are associated with type 2 diabetes. However, we did not find any association with diabetes-related phenotypes. Before concluding that the polymorphisms in NRF1 really are associated with the risk of type 2 diabetes, this result should be replicated.

At this time, we can only speculate on the mechanism linking genetic variations of $N R F 1$ to the susceptibility for type 2 diabetes. Although the nucleotide position $-46,350$

Table 3 Comparison of diabetes-related phenotypes according to haplotypes in the non-diabetic subjects

\begin{tabular}{|c|c|c|c|c|c|c|c|c|}
\hline \multirow{2}{*}{$\begin{array}{l}\text { Haplotype } \\
\text { combination }\end{array}$} & \multicolumn{3}{|l|}{ Haplotype $H 2$} & \multirow[t]{2}{*}{$p$ value $^{\mathrm{b}}$} & \multicolumn{3}{|l|}{ Haplotype $H 4$} & \multirow[t]{2}{*}{$p$ value $^{\mathrm{b}}$} \\
\hline & $-1-$ & $-/ H 2$ & $H 2 / H 2$ & & $-1-$ & $-/ H 4$ & $H 4 / H 4$ & \\
\hline Number & 264 & 38 & 1 & - & 180 & 109 & 14 & - \\
\hline HOMA-IR ${ }^{a}$ & $1.58(0.18-5.71)$ & $1.87(0.69-4.83)$ & 2.25 & 0.925 & $1.59(0.36-4.83)$ & $1.86(0.18-5.71)$ & $1.72(0.47-3.01)$ & 0.712 \\
\hline $\begin{array}{l}\text { Triglyceride } \\
(\mathrm{mmol} / \mathrm{l})^{\mathrm{a}}\end{array}$ & $1.36(0.38-5.94)$ & $1.51(0.55-2.87)$ & 1.79 & 0.805 & $1.44(0.38-4.63)$ & $1.51(0.52-5.94)$ & $1.46(0.75-2.41)$ & 0.330 \\
\hline $\begin{array}{l}\text { HDL-cholesterol } \\
(\mathrm{mmol} / \mathrm{l})\end{array}$ & $1.17 \pm 0.35$ & $1.19 \pm 0.28$ & 1.16 & 0.380 & $1.16 \pm 0.33$ & $1.19 \pm 0.36$ & $1.24 \pm 0.34$ & 0.298 \\
\hline BMI $\left(\mathrm{kg} / \mathrm{m}^{2}\right)$ & $23.5 \pm 3.2$ & $25.0 \pm 2.4$ & 23.7 & 0.013 & $23.6 \pm 3.2$ & $23.7 \pm 3.2$ & $23.5 \pm 2.8$ & 0.517 \\
\hline
\end{tabular}

Data are shown as means \pm SD in the case of normal distribution, otherwise as median (range). This table compares diabetes-related phenotypes according to the additive models with haplotypes $H 2$ and $H 4$, which showed a significant association with type 2 diabetes ${ }^{a}$ Homeostasis model assessment of insulin resistance (HOMA-IR) and triglyceride were log-transformed before analyses

${ }^{\mathrm{b}} p$ values of haplotype associations of additive models were calculated by the Haplo.Score algorithm developed by Schaid et al. [14], while controlling for age, sex and BMI as covariates. They were not corrected for multiple comparisons (seven parameters) 
was predicted as 3 '-flanking site of the $\mathrm{FOXCl}$ consensus sequence, haplotype analysis revealed that the $-46,350 \mathrm{~A}$ variant was neutral to the risk of type 2 diabetes in that it appeared both in protective (H2) and in susceptible $(H 4)$ haplotypes. Similarly, comparing the elements of $H 2$ and $H 4$, two polymorphisms $(g .+141 G>T$ and $g .+54529 A>G)$ were regarded as the determinants modifying the susceptibility to type 2 diabetes. However, their individual association with type 2 diabetes was only modest. Moreover, $g .+141 G>T$ is a synonymous substitution and $g .+54529 A>G$ is located in intron. It is possible that these polymorphisms did not reach a robust association with type 2 diabetes in this study because the sample size was too small to identify such a modest genetic effect. Since all genotyped loci were in a single LD block, it is unlikely that we failed to detect a disease association due to any polymorphism within this region that was not covered in genotyping. However, it is possible that the polymorphism(s) upstream from the promoter region sequenced by us or the gene(s) in the same or nearby locus of $N R F 1$ could have an influence on susceptibility to type 2 diabetes.

Type 2 diabetes is a multifactorial disease and influenced by gene-gene and gene-environment interactions [16-18]. Of note, there are interactions among $P G C 1, N R F 1$ and TFAM in the biogenesis and maintenance of normal function of mitochondria $[9,19,20]$. Recently, evaluation of high-order gene-gene interactions has become possible, thanks to newly developed methods such as multifactor dimensionality reduction [21-23]. The examination of gene-gene interactions among PGCl, NRF1 and TFAM would help reveal the genetic link between mitochondrial oxidative phosphorylation and type 2 diabetes.

In conclusion, haplotypes of the NRF1 gene, which regulates mitochondrial oxidative phosphorylation, are associated with type 2 diabetes in the Korean population and this association should be tested in other different populations for replication.

Acknowledgements Y.M. Cho and H.D. Shin contributed equally to this work. This work was supported by a grant from the Korea Health 21 R \& D Project, Ministry of Health \& Welfare, Republic of Korea (02-PJ1-PG1-CH04-0001 and 00-PJ3-PG6-GN07-001) and by an intramural grant of the National Institute of Health, Korea.

\section{References}

1. Petersen KF, Dufour S, Befroy D, Garcia R, Shulman GI (2004) Impaired mitochondrial activity in the insulin-resistant offspring of patients with type 2 diabetes. N Engl J Med 350:664-671

2. Petersen KF, Befroy D, Dufour S et al (2003) Mitochondrial dysfunction in the elderly: possible role in insulin resistance. Science 300:1140-1142

3. Gopalakrishnan L, Scarpulla RC (1995) Structure, expression, and chromosomal assignment of the human gene encoding nuclear respiratory factor 1 . J Biol Chem 270:18019-18025
4. Poyton RO, McEwen JE (1996) Crosstalk between nuclear and mitochondrial genomes. Annu Rev Biochem 65:563-607

5. Patti ME, Butte AJ, Crunkhorn S et al (2003) Coordinated reduction of genes of oxidative metabolism in humans with insulin resistance and diabetes: potential role of PGC1 and NRF1. Proc Natl Acad Sci U S A 100:8466-8471

6. Mootha VK, Lindgren CM, Eriksson KF et al (2003) PGC-1 alpha-responsive genes involved in oxidative phosphorylation are coordinately downregulated in human diabetes. Nat Genet $34: 267-273$

7. Evans MJ, Scarpulla RC (1989) Interaction of nuclear factors with multiple sites in the somatic cytochrome c promoter. Characterization of upstream NRF-1, ATF, and intron Sp1 recognition sequences. J Biol Chem 264:14361-14368

8. Evans MJ, Scarpulla RC (1990) NRF-1: a trans-activator of nuclear-encoded respiratory genes in animal cells. Genes Dev 4:1023-1034

9. Scarpulla RC (2002) Nuclear activators and coactivators in mammalian mitochondrial biogenesis. Biochim Biophys Acta 1576:1-14

10. Baar K, Wende AR, Jones TE et al (2002) Adaptations of skeletal muscle to exercise: rapid increase in the transcriptional coactivator PGC-1. FASEB J 16:1879-1886

11. Baar K, Song Z, Semenkovich CF et al (2003) Skeletal muscle overexpression of nuclear respiratory factor 1 increases glucose transport capacity. FASEB J 17:1666-1673

12. Eberle MA, Kruglyak L (2000) An analysis of strategies for discovery of single-nucleotide polymorphisms. Genet Epidemiol 19(Suppl 1):S29-S35

13. Alberti KG, Zimmet PZ (1998) Definition, diagnosis and classification of diabetes mellitus and its complications part 1: diagnosis and classification of diabetes mellitus provisional report of a WHO consultation. Diabet Med 15:539-553

14. Schaid DJ, Rowland CM, Tines DE, Jacobson RM, Poland GA (2002) Score tests for association between traits and haplotypes when linkage phase is ambiguous. Am J Hum Genet 70:425434

15. Quandt K, Frech K, Karas H, Wingender E, Werner T (1995) MatInd and MatInspector: new fast and versatile tools for detection of consensus matches in nucleotide sequence data. Nucleic Acids Res 23:4878-4884

16. Froguel P, Velho G (2001) Genetic determinants of type 2 diabetes. Recent Prog Horm Res 56:91-105

17. Elbein SC (2002) Perspective: the search for genes for type 2 diabetes in the post-genome era. Endocrinology 143:2012 2018

18. Gloyn AL, McCarthy MI (2001) The genetics of type 2 diabetes. Best Pract Res Clin Endocrinol Metab 15:293-308

19. Lowell BB, Spiegelman BM (2000) Towards a molecular understanding of adaptive thermogenesis. Nature 404:652-660

20. Wu Z, Puigserver P, Andersson U et al (1999) Mechanisms controlling mitochondrial biogenesis and respiration through the thermogenic coactivator PGC-1. Cell 98:115-124

21. Ritchie MD, Hahn LW, Roodi N et al (2001) Multifactordimensionality reduction reveals high-order interactions among estrogen-metabolism genes in sporadic breast cancer. Am J Hum Genet 69:138-147

22. Ritchie MD, Hahn LW, Moore JH (2003) Power of multifactor dimensionality reduction for detecting gene-gene interactions in the presence of genotyping error, missing data, phenocopy, and genetic heterogeneity. Genet Epidemiol 24:150-157

23. Cho YM, Ritchie MD, Moore JH et al (2004) Multifactordimensionality reduction shows a two-locus interaction associated with type 2 diabetes mellitus. Diabetologia 47:549-554 